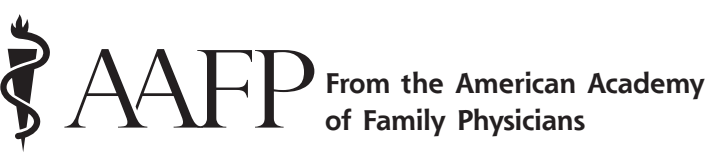

Ann Fam Med 2021;19:474-475. https://doi.org/10.1370/afm.2747.

\section{BUDGET NEUTRALITY UNDERCUTS PAYMENT IN 2022 PROPOSED MPFS}

A possible reduction in physician payment toplines a new AAFP summary of the proposed rule for the 2022 Medicare physician fee schedule (MPFS), available at https://www.aafp.org/dam/AAFP/ documents/advocacy/payment/medicare/feesched/ ES-2022MPFSProposedRule-072221.pdf, which the Centers for Medicare and Medicaid Services (CMS) released July 23, 2021.

The AAFP summary for members warns that a $1.6 \%$ reduction in allowed charges for family medicine could result if next year's fee schedule goes into effect as proposed without congressional intervention. Absent such a legislative remedy, "ongoing structural problems with the MPFS ... could result in many beneficiaries losing timely access to essential health care services," said the Academy and more than 100 other medical organizations in a July 23 letter, available at https://www.aafp.org/dam/AAFP/ documents/advocacy/payment/medicare/feesched/ LT-CongressionalLeadership-MPFS2022-072321.pdf, to House and Senate leaders.

\section{Payment}

The 2022 proposed conversion factor-the number multiplied by the relative value of each code in the fee schedule to determine the Medicare payment rate-is $\$ 33.58$, which is $3.75 \%$ lower than the 2021 conversion factor of $\$ 34.89$.

"This reduction can be attributed to the expiration of a $3.75 \%$ increase in the 2021 conversion factor, which Congress applied via legislation in December 2020," the summary says. Before lawmakers stepped in, however, the 2021 conversion factor reflected a reduction of more than $10 \%$ from 2020's rate, owing to a federally mandated budget-neutrality requirement working against the long-planned boost to evaluation and management coding for which the AAFP had strongly advocated.

\section{Telehealth}

CMS proposes to retain all services previously added to the Medicare telehealth services list on a temporary basis until the end of calendar year 2023. To implement the telehealth provisions in the Consolidated
Appropriations Act of 2021, the proposed rule also would, after the end of the COVID-19 public health emergency:

- remove geographic restrictions for telehealth services provided to diagnose, evaluate, or treat a mental health disorder;

- add a patient's home as a permissible originating site for telehealth services for the diagnosis, evaluation, or treatment of a mental health disorder $;$ and

- require, as a condition of payment for mental health telehealth services, that the billing practitioner must have furnished an in-person service to the beneficiary within the 6 -month period before the date of the telehealth service.

Audio-only telehealth services would be allowed for the diagnosis, evaluation, or treatment of a mental health disorder when:

- the originating site of the visit is the patient's home;

- real-time audio-video technology is available but the beneficiary lacks access, capacity, or willingness to use it; $_{;}$and

- an in-person service is furnished within 6 months of the audio-only service.

\section{Valuation of Specific Codes}

Among proposed new or revised valuations of current procedural terminology (CPT) and Healthcare Common Procedure Coding System (HCPCS) codes, the summary says, "of most interest to family physicians will be CMS's proposals related to the codes for chronic care management (CCM) and principal care management (PCM)." In those cases, the work relative value units (RVUs) and direct practice expense inputs recommended by the American Medical Association (AMA)/Specialty Society Relative Value Scale Update Committee for all these codes would be acceptedmeaning "an increase in the work RVUs for each of the existing CCM codes and 1 of the 2 PCM codes, which will be converting from HCPCS codes to CPT codes."

\section{Evaluation and Management Visits}

The Academy's summary notes a number of CMS policy changes proposed across 3 areas and pays particular attention to guidance related to visits split or shared by a physician and a nonphysician clinician working in the same group.

Among other updates, the 2022 MPFS would allow only the physician or nonphysician clinician who performs the substantive portion of the split or shared visit to bill for the visit ${ }_{i}$ "substantive portion" would be defined as more than one-half of the total time spent by the professional performing the visit. The rule would create a modifier to describe split or shared visits and to require that the modifier be appended to 
claims for such visits, regardless of which professional bills for the visit.

\section{Medicare Shared Savings Program}

In line with the AAFP's advice, CMS proposes to delay a previously finalized overhaul of quality reporting for Medicare Shared Savings Program accountable care organizations. CMS plans to extend the CMS Web Interface reporting option for 2 years and phase in the quality reporting changes over a longer period.

\section{Quality Payment Program}

The fee schedule also would make several changes to the traditional Merit-based Incentive Payment System (MIPS) track of the Quality Payment Program. The performance threshold would be 75 points in 2022, and the exceptional performance threshold would be 89 points. The cost category would be weighted at $30 \%$ of the MIPS score, and the rule would add 5 new episode-based cost measures, including 2 for chronic conditions. The quality, promoting interoperability, and improvement activities categories would be weighted at $30 \%, 25 \%$, and $15 \%$ percent, respectively.

\section{Academy Urges Correction}

To avoid "steep cuts" in payment to health care practitioners across the range of specialties, the Academy and scores of co-signatories called on Congress in their July 23 letter to maintain this year's 3.75\% conversionfactor increase through 2023.
More than 100 medical and health care societies, including the AMA and the Association of American Medical Colleges, signed the letter. It was sent to Senate Majority Leader Chuck Schumer, D-NY; Senate Minority Leader Mitch McConnell, R-KY, House Speaker Nancy Pelosi, D-Calif; and House Minority Leader Kevin McCarthy, R-Calif.

Medicare payments have been "under pressure from CMS' anti-inflationary payment policies for more than 20 years," the groups wrote, with the agency's annual updates to the conversion factor failing to keep pace with inflation. "The result is that the (conversion factor) today is only about $50 \%$ of what it would have been if it had been indexed to general inflation starting in 1998." At the same time, the letter adds, the cost of running a medical practice has increased by $37 \%$ since 2001-meaning a decline in physician pay of some $22 \%$ over that span when practice costs are adjusted for inflation.

"Moving forward, Congress must recognize the need for critical reforms to the MPFS system, including addressing the budget-neutrality requirement, which can lead to arbitrary reductions to reimbursement unrelated to the cost of providing care," the letter said. Without such change, patients suffer as clinicians "adjust to unpredictable and excessive reductions to reimbursement that inhibit their ability to ensure beneficiaries have access to the care they need-services that improve outcomes and lower costs."

AAFP News 